ÅRG. 6, NR. 1, 2017

ISSN (ONLINE) 2245-294X

Jessica Ruth Austin, Doctoral Researcher, Department of English, Film, Communication and Media, Anglia

Ruskin University, UK, jessica.austin@pgr.anglia.ac.uk

\title{
Online Hate and Hurt
}

\section{Ethical considerations when online research takes} an ugly turn

\begin{abstract}
When conducting online research, a researcher has an ethical obligation to safeguard the community and community members from any undue risk. In November 2014 David Kalac used an internet message board called 4Chan to upload pictures of a deceased woman who he had apparently strangled. In this case it was the users themselves who took the ethical responsibility to report the crime to the relevant authorities. The above is a clear cut case of possible crime that should have been reported by a researcher if they were studying them at the time, however other cases can be more problematic. In October 2015 a user on Tumblr named ZamiiO70 was bullied into attempting to commit suicide by members of the Steven Universe online community due to their consensus that her fan artwork was not "acceptable". In a study on the One Direction fandom, fans were seen threatening to throw acid in other's faces over a disagreement over Harry Style's new haircut. This paper will discuss the ethical dilemmas and challenges of researching an online community where there is a potential for harm in the real world and a discussion on how to mitigate these circumstances.
\end{abstract}

Keywords: research ethics, ethics, online research, trolling

\section{Introduction}

Internet research is becoming increasingly popular with scholars due to its convenience as a research platform and its plethora of hard to access groups. However, academics have lamented the policy gap when it comes to research ethics being still in its infancy (Warrell and Jacobsen, 2014, p. 24) and although debates are now beginning to stabilize with "certain understandings having become generally accepted" (Whiteman, 2012, p. 2) consensus among researchers on how to correctly implement these ethics when conducting internet research is still to be reached. Harm to subjects is the ethical focus of this article as although research ethics for academic institutions with their own codes of ethics will, and always have stressed that harm to the subject must be as minimal as possible it is not always clear how to do this when it comes to internet research. The very definition of "harm" when it comes to participants in research has also changed with time to now covering both physical and mental forms of harm and so development in the research process is still very much ongoing when it comes to ethics. That is not to say that discussions on internet research ethics have not paid attention to the researchers need to respond to "unexpected developments during the research process" (Whiteman, 2012, p. 13) but there 
have not been any clear guidelines to follow in response to these unexpected developments that may cause harm to subjects. This has meant that for the most part researchers have been left to try and deal with these situations in isolation and without clear guidelines which can be difficult for their own mental health; it is also unhelpful bureaucratically for the institutions where the research is being conducted as it becomes harder for an institution to approve and conduct studies as the ethics can be changeable with internet research.

This article will argue that ethics for internet research need to be situational rather than monolithic. Taking a universalist or monolithic stance where ethics must never be broken no matter what, the study can be damaging for research progress and so ethics should be more malleable and decided on a case to case basis (Goode, 1996; Whiteman, 2012; Estalella \& Ardevol, 2007). As the arguments for situational ethics have been argued far better elsewhere, this article will instead suggest ethical guidelines for a researcher for when their ethical stance may change due to coming across an unexpected development that was not foreseen in their original ethical stance. This article will also discuss the methodological difficulties of studying bad behaviour online in the context of the discipline of fan studies. Current work in the field is beginning to become concerned with the difficulties of how to establish whether comments taken from fans within the online communities are from the fans themselves or those who just want to bully or "troll". This is important to discuss as it can create a situation where the researcher is representing bad behaviour from outside of the community as coming from within it, leading to biased and potentially incorrect data. The purpose of this article is to specifically discuss the research of trolling and bullying in particular, rather than grand scale internet research and will do so by suggesting a simple guideline which researchers can follow if they come across a bullying scenario in their research. The guideline includes 7 points which details behaviour that can occur in a bullying event that may require their attention ethically and then gives examples of how to combat these issues. There will be reference to the case of Zamii070 in the Steven Universe fan community and the ethical considerations emerging from such a case.

\section{Discussions on Ethics so far}

In Kozinets Netnography (2010) and in his previous works (2002; 2006) and in Whiteman's Undoing Ethics (2012) they describe the main arguments that have occurred when it comes to establishing research ethics in internet studies: privacy of data and legality, whether data collected is from human subjects and participation vs. concealment. Although these have been well argued by researchers over the years this researcher has found there to be a lack of emphasis in each of these debates surrounding the concept of harm. Often the onus is on the researcher to avoid these issues rather than being reactive in cases where these issues become a problem; Schrag (2011) attributes to social science research in the U.S in particular being "rife with misconceptions, inconsistencies in decision making, and mission creep in the oversight by IRBs" which echoes other complaints that increasing bureaucracy by institutions have limited research (Wiles et al., 2010, p. 2; Whiteman, 2012, p. 8) with the main arguments detailed as follows.

\section{Privacy}

The concept of what is public and what is private when it comes to internet research is a difficult issue and quite rightly has been debated vigorously by academics according to Kozinets (2010, p. 140). The concept of what is public and private has certainly changed in debate and many practices in early internet research argued that the internet was merely text (Kozinets, 2010, p. 141) and so did not require an ethical stance at all as it could potentially be read by anyone; As the way people have used the internet has changed however, websites or forums that require membership has meant that only members can see or exchange messages which has brought forth questions on whether this would allow people a "degree" of privacy (Bryman, 2012, p. 681). For Hewson et al. (2003), comments posted online are in the public domain and there is an expectation by the commenter that it will be read and so there is no need for informed consent. However, many commentators do not even think about the implication of their words being used for research purposes especially by those outside of their internet community and can sometimes react angrily to this (Kozinets, 2010, p. 141). For Zimmer (2010) on social media sites in particular, the commentator has an expectation that their comments will only be read contextually for the directed audience i.e. other users and not for research purposes which would then suggest that ethically speaking these comments although online are actually private. Although there are pros and cons for each 
argument it is realised that there is not a clearly recognizable binary that clearly distinguishes the public and private in this type of research (AOIR, 2012, p. 7). Though anonymity is often preferred in research it can be very difficult for researchers to keep the data confidential as even anonymised data could contain identifiable information such as origin of the computer generated message and personal information (Bryman, 2012, p. 680; Stewart and Williams, 2005; AOIR, 2012, p. 7). This is caused by the fact that unlike in more traditional data collection such as questionnaires or interviews where the data is seen and held by the researcher only, data found online can potentially be seen by everybody.

Criminality should also be considered when researching certain comments. For the UK in particular there have been a spate of investigations by police of hateful comments online such as arrests following threats of rape directed towards Caroline Criado-Perez (BBC News, 2013). As well as this in the United States, an investigation and arrest was made when a girl convinced her boyfriend to commit suicide via text (Metro, 2016). When conducting research online unfortunately a researcher may have to investigate abusive comments in general and also that directed at other users and this can create an ethical problem when it comes to privacy depending on the laws where the researcher is located, and which website the abuse is occurring on. There is a lack of literature with advice for these situations which is why a possible guideline for this is provided later in this paper.

\section{Human Subjects}

Rimm (1995) is a now infamous study on pornographic material on Usenet which had clear ethical violations when it came to its subjects, suggesting that "those who blocked access to their files might be child molesters; and the suggestion in a resulting grant application that the research might assist in the prosecution of users in helping to 'identify and prosecute Rimm's research subjects'" (Whiteman, 2012, p. 7). Due to information being obtained by lurking and access to non-public computers Rimm did not consider the real life impact on his subjects. This is not to say that researchers and institutions have still wilfully ignored human subjects within internet research; it is easy to classify working with human subjects in a face to face interview but when a researcher is looking at what someone has written over a computer screen it is a little more difficult. The AOIR released an ethics guide for internet research in 2002 and updated it in 2012 and address this very issue stating that "human subject has never been a good fit for describing many internet based environments" $(2012$, p. 6), especially as lots of studies on the internet never speak to the participants in real time when it comes to research such as qualitative analysis of forum comments. With internet research in particular it is difficult to control the environment of research as opposed to face to face interviews or focus groups and so it makes it even more likely that ethical quandaries will occur; in a face to face interview a researcher knows exactly who they are talking to whereas the internet gives a user the opportunity not only for anonymity but also to create a fake persona. This means that in the online environment the subject may not even know who is bullying them which can cause more paranoia and emotional distress and means that it is harder for a researcher to proceed when dealing with this bullying; in a face-to-face scenario such as research in a school a researcher could identify the subjects involved and get them each the help they need to tackle the bullying. This means that it is harder to implement ethics to deal with this behaviour and a prime example of why ethics need to be applied on a situational basis in online research.

Susannah Stern is a classic example of where research ethics did not go far enough to protect human subjects. In her study examining personal websites of young people (2004) she was advised by her institution that her study did not involve human subjects and so an ethical review was not required. During her research she came across websites that indicated that one of the young owners wanted to kill themselves, due to the advice of the ethics board she did not follow up on the owner of the comments and sometime after the adolescent did indeed commit suicide (Whiteman, 2012, p. 40). If this research had been conducted with the adolescents face to face there would have been an ethical responsibility to follow up on this subject in danger of harming themselves, though of course this may still have not prevented suicide in any case but would have at least provided extra support.

\section{Participation}


How much of a participant the researcher becomes in conducting internet research can be ethically and sometimes methodologically problematic. In fan studies there has been much discussion on this issue with Matt Hills creating the category of Scholar Fan (or Aca-fan) to describe academics who wear their fandom in public (Hills, 2002) in that the academic is studying a fandom in which they have a personal interest. Although this can be useful in terms of already being familiar with the nuances of the community being studied and can be helpful in terms of gaining access to the community it means that some fans "go into the research process with the aim of legitimating and representing their own communities (Duffett, 2013, p. 263) and for Henry Jenkins "writing as a fan means as well that I feel a high degree of responsibility and accountability to the groups being discussed" (Jenkins, 1992, p. 7). This means there is a very real danger of the researcher "over-identifying with the setting and, in so doing, losing their research perspective" (Whiteman, 2012, p. 112).

However there are ethical problems too with "lurking", a term that refers to total non-participation in an online research situation and recording data without the participants being aware. In a discussion with a colleague, Whiteman compared this way of research to the act of spying in the offline world as offline researching in this way would often require taken a covert or deceptive position (Whiteman, 2012, pp. 109-112). For Whiteman it made her reflect on what it meant to be specifically an academic lurker, rather than a lurker for non-academic activities in "that the interests of researcher and researched are not the same." (Whiteman, 2012, p. 118).

There are also methodological problems that relate back to ethics when it comes to researching internet groups when trolling and harm are concerned. A researcher should be careful not to represent members of an online community as bullying other members if in fact the trolls are not members of the fandom at all but merely posing as such as in some cases it is not obvious. Currently there are few methodological guidelines on this and it seems that most researchers are just told to use a "common sense" approach, but what may seem like the correct method to one researcher may not be the same for another leading to discrepancies between studies.

\section{\#BlackStormTrooper}

On November 28th 2014 the first trailer for the upcoming Star Wars: The Force Awakens debuted and within a few hours began trending on the social media platform Twitter. However there was great distress when media outlets began to report that \#BlackStormTrooper was trending and how the majority of tweets were from fans who were being racist about the black actor John Boyega, who plays a Stormtrooper in the movie. In a study using discourse analysis by William Proctor however it seems that many of these tweets sent under the \#BlackStormTrooper hashtag were actually condemning racism (though some of those were condemning it using violent language) and that many were actually questioning the canonicity of having a black male as a Stormtrooper as these soldiers in canon are supposedly clones of one man, Jango Fett (Proctor, forthcoming). Proctor discusses that from the few instances that he discovered that were racist in content directed to the actor he was unable to be sure whether they were a troll or an actual member of the Star Wars fandom due to the use of online pseudonyms. Therefore this researcher argues that it would be incorrect and unethical to state that Star Wars is a racist fandom or even that it is suffering from a racism problem because methodologically, in this study at least, it cannot be proven that these people come from the fandom community, or, indeed, if they even exist at all, at least on the hashtag in question.

Other incidents which occur due to the anonymity of the internet make not only internet research sometimes more difficult than offline research but can also make internet research ethics harder too. In October 2012 a tweet seemingly coming from the verified Twitter account Entertainment Weekly announced that popular music star Justin Bieber was suffering from cancer spurred the trending of the hashtag \#BaldforBieber; it was later revealed to be a hoax perpetrated by online message board 4Chan (MashableUK, p. 2012). What makes this case difficult for a researcher is that it is unclear whether anyone actually fell for the prank which encouraged Bieber fans to shave their head in solidarity with him as several of the pictures and videos of those going bald are photoshopped and edited. As well as this in a study of One Direction Fans there were tweets from supposed fans of the boy band who were threatening to throw acid in the face of the editor of $G Q$ for a supposed slight towards the band and suggestion of other violent behaviour, most notably because one fan did not agree with the opinion 
of another on the haircut of one of the band members, Harry Styles (Proctor, 2016; Jones, 2016). Once again it can make it difficult for researchers to know whether the abuse is coming from actual members of the community and also makes it difficult to safeguard those in the community in which they are studying as it can be difficult to know whether these threats are serious and which will lead to real life crime.

Often researchers have been left with little advice on how to deal with threats to users and often have to resort to simply doing nothing due to the methodological challenges and the lack of help from ethics boards or institutions. Although some incidents of trolling do not seem to actively hurt other users other times online bullying has affected the offline lives of those involved.

\section{Social Media Studies}

It is not just trolling by others that can cause emotional distress to a participant but also the action of the researchers themselves as in the case of research carried out on users of Facebook. In 2012 a study was carried out on users which used post manipulation (hiding or promoting of certain posts) to examine the effect of emotion contagions - how emotions can be affected and filtered by social media use (Kramer et al., 2012). However there was a huge backlash to this from the users with many arguing that even though they had given their consent to be research participants as part of Facebook's terms and conditions they still believed they had not given informed consent. As well as this many of the groups targeted in the research were considered vulnerable. The publishers of the piece, PNAS, released this statement in response to the public backlash against the research clarifying that:

As a private company Facebook was under no obligation to conform to the provisions of the Common Rule when it collected the data used by the authors, and the Common Rule does not preclude their use of the data (PNAS, 2014)

As the Common Rule being the protection of human subjects as stated by the Office of Human Research Protections (HHS.GOV, 2017) did not apply this research was deemed as ethically sound from a regulator's point of view. One of the editors of the study, Susan Fiske, did raise questions of the ethics of the research and lamented over whether it was socially acceptable from an ethics standpoint. (The Atlantic, 2014). Facebook found itself defending its research again in 2017 when The Australian found a 23 page document leaked from Facebook which "outlines how the social network can target "moments when young people need a confidence boost" (The Australian, 2017). Although this may seem like good intentions, targeting young people, especially those under the age of 18 can be ethically misguided and provide more harm than good. There has been a previous study that looked at how social contagion can be an influence in self-harm and presented approaches to minimise the social contagion (Richardson et al., 2012, p. 122) which also has good intentions, but raises the question of how much intervention a researcher should be doing.

Therefore it is important for a researcher to really consider what kind of online research they are doing and what ethical stance they take on intervention in these cases. The guideline that is suggested later works best with academic scholars who are working with young people and children online as although there are rigorous ethical protocols for researching under $18 \mathrm{~s}$ offline, there are little guidelines on how to safeguard children in research online. Therefore there should be more ethical safeguards when it comes to vulnerable groups and young people when it comes to internet research because as Fiske pointed out, ethics are usually socially acceptable for the current time and it is clear from public reaction to research on vulnerable people that the public require more regulation and consent to accept the research as ethical. Furthermore an ethical guideline would be in keeping with current recommendations for safe internet use in children and young people such as from organisations like the UK Council for Child Internet Safety (2017). For Max Masnick, a researcher with a doctorate in epidemiology:

As a researcher, you don't get an ethical free pass because a user checked a box next to a link to a website's terms of use. The researcher is responsible for making sure all participants are properly consented. (The Guardian, 2014) 
The guideline that is suggested at the end of this paper then, is most useful then when researching communities which may have young or vulnerable members as it adds extra safeguarding for these participants. As well as this it is most useful when working with communities rather than as a lurker or covert researcher as the guidelines can be used in conjunction with the rules of conduct and terms and conditions of the website which is hosting your research.

\section{Zamii070, Homestuck and Steven Universe}

The following case study was chosen because unfortunately it shows a worst case scenario and easy to follow timeline of events for online bullying. As well as this due to members of the communities archival of posts from the incident there is lots of data concerning what was posted at the time which are still readily available online; in other cases of online bullying posts are quickly deleted by those involved making it difficult to analyse what happened at the time forcing researchers to use secondary accounts. Also, the abuse in this case happened solely online whereas other cases which have been publicised in the mainstream media have happened to the subject at school as well as the home. This is important because some have viewed online bullying as having less of a psychological impact on subjects than offline bullying. However research suggests that online bullying can cause more psychological distress than school bullying (Schneider et al., 2012, p. 174) and the effects can differ between genders (Nordahl et al., 2013, p. 394) with girls being more likely to suffer cyberbullying (Schneider et al.,2012, p. 173).

\section{What Happened?}

Zamii070 was a fan artist who often shared her artwork on the social media blogging website Tumblr from various different shows, having drawn art primarily of Steven Universe (SU) characters but also Yowamushi Pedal (Japanese Anime), Homestuck and My Little Pony (MLP). In the weeks following there were several users who began to comment on Zamii070's artwork in a negative way; For example, claiming that her drawing of FlutterShy (MLP) as a Native American was cultural appropriation, her drawing of a slimmed down RoseQuartz (SU) was fat phobic and that by redrawing some artwork which was commissioned by someone who turned out to be a convicted paedophile after the fact meant that she supported paedophilia and was a paedophile herself (Imgur, 2015). This in turn was followed by a "receipt blog" creation on an account on Tumblr where all her apparent transgressions were detailed and would regularly comment on her personal account. A receipt blog is usually targeting one user and the purpose of these blogs is for people to keep the evidence "of wrongdoing" for future reference even if they have been deleted from the targets account. Over 40 critical blogs were created seemingly by fans of either SU or Homestuck to also send her critical messages (Daily Dot, 2015). Coupled with rumours of issues at home and the constant bullying from the hate blogs directed towards her on the $20^{\text {th }}$ October 2015 Zamii070 left a final message on her Tumblr account insinuating that she was going to kill herself and then did not post for 3 days causing worry in the community. After 3 days Zamii070 returned streaming a feed from the hospital where she was being treated after her suicide attempt to let her followers know that she was indeed still alive.

\section{The Fallout in the Community}

Within the SU community there were various postings from members in support of Zamii070 and threads of encouragement with many fans condemning the abuse she was receiving and a year on the fallout from the Zamii070 incident is still felt in the community. In posts that have appeared on the $\mathrm{r} /$ stevenuniverse forum on Reddit and discussing the situation now many users felt that a small portion of the fandom was still toxic and bullying members; Lauren Zuke, a writer for SU felt that she needed to quit Twitter after receiving abuse from some fans due to her involvement in the episode "Beta" which seemed to confirm a relationship between two characters that some fans did not like (Gizmodo, 2016). Furthermore, moderators on the r/stevenuniverse forum have repeatedly made very clear that they heavily policed any threads which discussed the Zamii070 incident for any bad behaviour and would be deleting any derogatory comments. Unfortunately for the Steven Universe community in particular there were articles in the mainstream media that labelled the fandom as toxic and 
therefore has decreased the reputation of the fandom to those outside the community even a year after the incident.

\section{Ethical Considerations}

To research a case such as Zamii070's is sadly not going to be an uncommon occurrence as researchers delve further into online communities. This researcher became interested in the Steven Universe community after the events of Zamii070 had already occurred and so did not see events as they unfolded but this gave this researcher a chance to reflect on what they would have done if they had been researching the community at the time.

One of the pertinent points to consider in this case was the receipt blog that was hosted on Tumb/r which hosted Zamii070's artwork on the page specifically to allow users to comment negatively upon them; it also allowed users to redirect from this page onto Zamii070's own Tumblr blog and so made it much easier for them to send her hateful messages directly. This continued for a sustained period even though it went against Tumblr's terms of service for harassment which specify that users not only do not "Engage in targeted abuse or harassment" but also that they must not "attempt to circumvent the block feature or otherwise try to communicate with them. Just stop." If the user themselves has blocked them to try to stop the harassment (Tumblr, 2016). However, as with any website, especially on the scale of Tumblr, which has millions of users, moderation can be very difficult and time consuming and so for Zamii070 the abuse was able to continue even though Zamii070 was clearly being harassed. On smaller websites and forums such as the Reddit subpages dedicated to r/Homestuck (one of the fandoms Zami070 drew artwork for) and $r$ /StevenUniverse moderation is much easier as the moderators only have to focus on submissions from one place rather than across a whole website making it far easier to ban harassing users and safeguard their members. Therefore it may be easier ethically for researchers to conduct their research on smaller websites rather than across whole platforms as it will take strain off the researcher when it comes to safeguarding their human subjects or those they are studying covertly as moderation of harassment is quicker in response.

Another point to consider in the Zamii070 case is that the bullying emanated from a minority corpus but rapidly multiplied into a group or "mob". Although this researcher cannot be sure of the actual number as theoretically one user could create multiple accounts to post harassment from, to Zamii070 at least, it would seem as though more and more people were bullying her and consequently made her feel worse as her posts indicated. Although many of the original blog posts harassing Zamii070 have since been deleted by their users an archive of much of the abuse she received is still available online with multiple accounts on record as sending the abuse, the link to this archive will not be recorded here. This is because like with former abused children, knowing that there is a record of the abuse for others to see online can be very traumatic (Taylor and Quayle, 2003, p. 204) and therefore it would be inappropriate for it to be linked here especially as the subject of this research is about minimising harm.

For a researcher this must be an ethical consideration as the bullying clearly progressed in a damaging way for Zamii070. If it was just one abuser then the bullying would not have been as severe and a potential researcher may not have decided to intervene initially. This raises an interesting ethical concern for researching trolling in general, if trolls are targeting several accounts that the researcher is researching compared to concentrating on trolling one particular account what safeguards should there be? Should there be a difference in the way that these trolling incidents are handled? For this researcher and the Zamii070 case as trolls were concentrating on one victim and it was clearly having an emotional toll on the user, then ethically a researcher should take steps as part of safeguarding that particular human subject.

Consideration must also be made to how these incidents should be researched after the event has occurred. In researching this event many users were cautious to speak about the Zamii070 incident as opposed to Steven Universe in general, some due to worry that being critical of Zamii070 would make them seem like a bully, others due to feeling it was inappropriate to comment as it had led to her trying to kill herself, causing both ethical and methodological problems as well. In some instances it will be far more appropriate to look at the secondary 
sources of archived material such as the Zamii070 hate archive material than it would to contact Zamii070 directly for her experiences. This may cause issues with research on the effects of trolling as it may be impossible to get primary sources but ethically it could conflict with safeguarding the participant welfare as answering research questions on the subject may be too much for the participant to emotionally handle. Therefore caution must be used and researchers must clearly show that ethically the benefits of the research will outweigh the negatives of such experiences.

\section{Improving Ethical Research Protocols}

One of the main issues that researchers face is that there are few guidelines or agreements for appropriate and ethical behaviour when it comes to this kind of research. The internet has developed a huge amount in the ways that many people use it with social media, online shopping, online gaming all on the rise and ethical research is finding it difficult to keep up, especially with the explosion of social media and the sheer amount of social media users. In this section this paper is going to suggest a guideline on what steps to take in a case such as online bullying so as to remain ethical and to try and establish a framework of guidelines that can be used by most online researchers. There are no doubt pros and cons to suggesting a new guideline for dealing with online bullying and trolling. One of the major difficulties with the suggested guideline is that often it will mean extra work for the researcher themselves and the fact that they may be potentially revealing themselves as a researcher could prove problematic for anyone conducting covert internet research. Unfortunately there can never be a real consensus when it comes to ethics as researchers will always differ on what they deem to be ethically necessary however a guideline with suggestions on what behaviour that the researcher may see during their research may be able to guide researchers on what action could be appropriate to take. It is important to note that this guideline is aimed at researching young and vulnerable people online; although celebrities are often targeted by online abuse often they have PR teams, agents and supportive entourages who monitor their social media and are readily available to deal with any trauma, they are also less likely to see abusive messages as these are usually dealt with by their PR team compared to someone with a personal account.

One of the first and most important things a researcher has to do to implement appropriate research ethics is to identify whether their communities members exhibit risky behaviour which can encourage online bullying. If their participants are young and are engaging in the 9 risk factors in a Harvard medical letter such as "Interacting with strangers" or "Making rude or insulting comments to someone else online" (Harvard Health Publications, 2008) the researcher is more likely to observe harmful behaviour and so will need to protect themselves more thoroughly from an ethical standpoint. These risk factors are more likely to be found on an open social media network such as Twitter rather than a virtual game website such as Neopets which although aimed at children and young people has much less person to person interaction; the proposed guidelines may be more helpful for studying these groups on social media. As well as increased risk taking, researchers should identify whether the communities are more at risk of harming themselves to begin with, such as evidence of self-harm in the online study of the LGBT community McDermott et al. (2013). This study is interesting ethically because the only explanation in their ethics section of whether covertly researching their participants could lead to harm was that the three authors had "experience of utilising online research methods for hard-to-reach populations and sensitive subjects" (2013, p. 128). This suggests to this researcher that they were following their "common sense" rather than ethical reasoning and also ethically echoes the Facebook study which was publically denounced as unethical because the participants were vulnerable. Therefore if the researcher can identify their group as young and/or vulnerable they should use the guideline suggested in this paper so as to not just having to rely on past experience as a researcher which has not gone down well as an ethical protocol with the public.

There are some commonalities in behaviour seen online that may suggest a human subject is in danger and so the guideline can be made flexible for different cases but without the need for a researcher to solely rely on their own "common sense" as they have been left to so far. Research from the psychological discipline has identified common signs of when someone may be suicidal or depressed for instance which can be extrapolated from an online context. Such as in the Zamii070 case many people will actively tell people that they want to die or that life isn't worth living (Grohol, 2009), will become obviously agitated and even give away their possessions (Your 
Life Counts, 2017). Therefore a researcher does not have to rely on their own subjective opinion of the situation but can use the warning signs as set out by the American Psychological Association (2017) or their own national society.

\section{Guideline for safeguarding research participants}

1. Has the subject stated that they are upset about any abuse they have received?

2. Is the subject being bullied by multiple accounts/users

3. Has the bullying been occurring for a period of time?

4. Has the bullying progressed over time?

5. Has the subject alluded to the fact that they may harm themselves?

6. Has the subject stated overtly that they will harm themselves and intend to do so?

7. Is the subject being subjected to extreme threats such as murder and do these threats contain intent?

The above points should be seen as a red flag for any researcher but does not mean that a researcher has to reveal themselves as such if they are doing covert research, which may be a worry for those trying to research and remain ethical. Depending on the answers given by the researcher in response to the guidelines the following steps should be taken below.

If the researcher has answered yes to any point between 1 and 5 they should proceed to do the following:

- Report the behaviour to the host website

On Tumblr for example, the support part of their website allows users to report behaviour that breaks their terms of service such as abusive behaviour and even allows the user to upload screenshots of the behaviour just in case it is deleted by the perpetrators later. On Reddit, a researcher can email the moderators of sub-reddits such as $r / S t e v e n U n i v e r s e$ directly without the need to reveal research status and would still allow the researcher to carry on researching the community knowing that they have done their safeguarding duty by reporting the behaviour to those who essentially have the power to stop the behaviour.

\section{- Monitor Progress}

If the same accounts are still harassing the user then the researcher should submit a new report to the host website. The researcher should then make a diary entry and screenshot of any behaviour after their report to the host website as many of the larger websites such as Twitter can use this evidence to close down any offending accounts.

For the first 5 guidelines these two steps are the only ones required from a researcher and would cover them ethically as they have taken appropriate action in reporting online abuse and harassment, however if either point 6 or point 7 has occurred or has progressed to this stage from the researchers monitoring of progress then it is the researcher's ethical responsibility to respond and respond quickly. These behaviour points could have a potential real life, real world consequence for the participant and thus should be treated as harm for the participant when it comes to research ethics.

If the researcher has answered yes to either point 6 or 7 and they suspect the participant to be under the age of 18 they should proceed to do the following:

- Report the behaviour to the local authority

In situations where the threats have become extreme and the researcher has already notified the host website then a report should be made to the local law enforcement. If it can be easily ascertained where the human subject is located then a report to the subjects own local law enforcement rather than one in their general area.

- Archiving of Evidence 
Using the diary entries and screenshots that the researcher has compiled due to monitoring the progress of the abuse these should then be compiled in an archive for retrieval at a later date by law enforcement if needed. This is needed in case the online abusers delete their accounts.

\section{- Revealing Researcher Status}

This is an optional last resort if the researcher suspects that a research subject is imminently in danger from others or themselves. Due to the graveness of the situation, especially if the human subject has stated overtly thoughts on killing themselves then this may be a point where a researcher may feel the need to reveal themselves to the user and/or the community if they feel that urgent help is needed.

\section{- Ending of Research}

This is also an optional point which may occur if the researcher has had to or chosen to reveal themselves to the community. For a researcher's own protection it may be prudent to withdraw from the research environment as they may be targeted by trolls or abusers themselves for reporting the extreme harassment.

The guideline has been suggested because there seems to be a lack of literature to help researchers identify problematic behaviour which could affect their subjects when it comes to harm. This guideline has also been suggested because in many instances a quick email will suffice to be ethically covered when it comes to safeguarding their participants whether overtly or covertly studied and so can be dealt with quickly. For researchers being able to defend their work ethically has become more important due to controversial research in the past and so having another point in which to say they have safeguarded their participants in their ethical review will be most welcome to many researchers.

Even with this guideline however there are still points to consider. It must be remembered that intervening within a community is not ethically responsible in some cases and that sometimes research participants may not want this; in a study on adults with intellectual disabilities the participants felt more threatened and saw it as more harmful if researchers needed to report information to the police, such as if they were being hurt (McDonald et al., 2017, p. 83). A researcher then has to balance their ethical responsibility with the needs or wants of the research participant when it comes to intervention. To counter this point then this researcher would argue that following the preliminary guidelines for in response to points $1-5$ which are to report to the hosts website and monitor progress are not intervening. This is because on the websites that these guidelines have been suggested for e.g. social media websites, there is often a code of conduct that encourages users to report bad behaviour. In online communities and chatrooms there are also, even if they are unofficial, standards of behaviour that often tie in with encouraging reporting of bad behaviour. As such it can be said that the researcher is following the standards expected by the host website and as it is a function that is available to any member of the website that reporting a situation is not going above what is expected from normal members. If points 6 or 7 occur then this guideline recommends contacting the local authorities which would be intervention however. Therefore this guideline suggests this only for participants who are under the age of 18 . This is because from a theoretical ethics standpoint in offline research far more safeguarding protocols are in place for researching under $18 \mathrm{~s}$ (for good reason) and so this should be replicated in the online environment. Furthermore, other studies have shown that intervening in a positive way on social media has had positive effects on youths at risk of suicide (Rice et al, 2016) and that the use of "real time monitoring" can flag up people in crisis and are "important for overcoming difficulties mental health services have with responding to fluctuating SI [Suicidal Ideation] and episodes of SH [Self-harm] and SA [Suicide Attempt]" (Cox and Hetrick, 2017).

\section{Limitations}

The researcher must make sure that they are aware of the social protocols of the community before they implement these guidelines; slight trolling in the community 4Chan, for example, is expected and often encouraged. Therefore these guidelines are most helpful to those who already know their community well enough to know the learned behaviours such as with scholar fans. As well as to also not be considered as ethicslite this guideline is best used where the researcher is doing non-covert research alongside the community. 
These guidelines are not entirely based on ethical theoretical perspectives but instead on analysis that has occurred in psychological studies and the ethical reactions to studies that have been conducted on vulnerable groups (Facebook). Although this may seem like a weakness there is an obvious need to look at these positions due to public backlash. Due to the scope of discussion in this paper the guideline does not offer a way to combat either trolls/ or deal with those using fake personas. However, it is important to be able to come up with ways of ethically researching those who are being online bullied as online bullying is unlikely to stop in the near future despite implementations from anti-bullying groups and agencies. Harm and intervention are still being debated in scholarship and it may be shown in later research that these guidelines are not enough or have created harm as described by Maheu et al:

In coming years, there are likely to be many instances in which professional intervention in an emergency from a distance has succeeded and others in which it has resulted in harm to the practitioner or made matters worse for the patient (Maheu et al. 2004, p. 259)

However this doesn't mean that we should stop trying to be more ethical as we may learn from our mistakes such as researchers did when it came to the aftermath of Stern's study.

\section{Conclusion}

One of the main points that this researcher sees as problematic when it comes to previous internet research is that those being researched have sometimes not been seen as human subjects which should definitely not be the case. A researcher should be conscious of the fact that comments are written by real people with real feelings and that can sometimes be forgotten when they are looking through a computer screen. Researchers should be very wary of this, especially when conducting research in "real time" such as social media, as there have been claims that some young people have taken their lives due to the internet abuse they have suffered. Cyberbullying has been blamed for a plethora of young people who in the recent past have committed suicide or tried to commit suicide due to trolls with now famous victims in the USA such as Amanda Todd, Megan Meir and Jessica Logan.

What is important to note however is that it is not a researcher's fault nor responsibility to stop these terrible tragedies happening as that would be putting too much onus on the researcher. However just as with offline research ethics the researcher should put great importance on safeguarding, which as shown in this paper, can be far harder to do in online research. This researcher decided not to put too much detail into the covert/overt argument in internet research as safeguarding does not necessarily mean that the researcher has to reveal themselves as such even when safeguarding their human subjects. In many instances an anonymous email to moderators is all a researcher has to do to have ethically protected their human subjects; even if abuse provokes a user into trying to take their own life at least the researcher would have acted and so would hopefully not be subject to the bad feelings experienced by Susannah Stern and others when they have not been advised as to the "human" status of their participants.

To conclude, internet research ethics is an ever changing and incredibly dynamic area which is undergoing changes all the time, along with the changes that the internet has undergone over the years. With the advent of social media and the increasing interconnectivity of people online there is a huge amount of different ways to research and how to do this ethically. This researcher would like to think that their short guideline will be helpful to those who are studying online communities when researching takes an ugly turn for the worst and may have far reaching consequences that extend beyond the online sphere.

\section{References}

American Psychological Association (2017). Suicide Warning Signs: Learn how to recognise the danger signals [article], http://www.apa.org/topics/suicide/signs.aspx

AOIR (2012, December). Ethical Decision-Making and Internet Research: Recommendations from the AoIR Ethics Working Committee (Version 2.0), [guidelines], https://aoir.org/reports/ethics2.pdf 
The Atlantic (2014, June 28). Even the Editor of Facebook's Mood Study Thought It Was Creepy, [article], https://www.theatlantic.com/technology/archive/2014/06/even-the-editor-of-facebooks-mood-studythought-it-was-creepy/373649/

The Australian (2017, May 1). Facebook targets 'insecure' young people, [article] http://www.theaustralian.com.au/business/media/digital/facebook-targets-insecure-young-people-to-sellads/news-story/a89949ad016eee7d7a61c3c30c909fa6

BBC News (2013, July 29). Caroline Criado-Perez Twitter abuse case leads to arrest, [article], http://www.bbc.co.uk/news/uk-23485610

Bryman, A. (2012). Social Research Methods, Oxford: Oxford University Press

Cox, G. \& Hetrick, S. (2017). Psychosocial interventions for self-harm, suicidal ideation and suicide attempt in children and young people: What? How? Who? and Where? Evidence-Based Mental Health, 20, 35-40.

Daily Dot, (2015, October 27). 'Steven Universe' fandom is melting down after bullied fanartist attempts suicide, [article], http://www.dailydot.com/parsec/steven-universe-fanartist-bullied-controversy/

Duffett, M. (2013). Understanding Fandom: An Introduction to the Study of Media Fan Culture, New York/London: Bloomsbury Publishing Plc

The Guardian (2014, June 30). Facebook emotion study breached ethical guidelines, researchers say, [article] https://www.theguardian.com/technology/2014/jun/30/facebook-emotion-study-breached-ethical-guidelinesresearchers-say

Gizmodo, (2016, August 13). Steven Universe Artist Quits Twitter Over Fan Harassment, [article], http://io9.gizmodo.com/steven-universe-artist-quits-twitter-over-fan-harassmen-1785242762

Goode, E. (1996). The Ethics of Deception in Social Research: A Case Study, Qualitative Sociology, 19, 11 - 33

Grohol, J. (2009). Common Signs of Someone Who May be Suicidal. [blog post], https://psychcentral.com/blog/archives/2007/10/08/common-signs-of-someone-who-may-be-suicidal/

Estalella, A. \& Ardevol, E. (2007). Field Ethics: Towards an Ethics Located for the Ethnographic Research of the Internet, Qualitative Social Research, 8(3), Forum: Qualitative Social Research, 01 September 2007, Vol.8(3).

Harvard Health Publications (2008). Protecting children and teens from cyber-harm, Harvard Health Publications. The Harvard Mental Health Letter, Harvard Health Publications. The Harvard Mental Health Letter, Jul 2008.

Hewson, C., Yule, P., Laurent, D. \& Vogel, C. (2003). Internet Research Methods: A Practical Guide for the Social and Behavioural Sciences, London: Sage

Hills, M. (2002). Fan Cultures, London: Routledge

HHS.GOV (2017). Federal Policy for the Protection of Human Subjects ('Common Rule'), [guidelines] Office for Human Research Protections: U.S Department of Health and Human Services, https://www.hhs.gov/ohrp/regulations-and-policy/regulations/common-rule/index.html

Imgur, (2015, October 25). I pulled together a bunch of posts that show what exactly led up to the suicide attempt of tumblr artist Zamii070., [blog post], https://imgur.com/a/USROb

Jenkins, H. (1992). Textual Poachers: Television fans and participatory culture, USA: Routledge 
Jones, B. (2016). "'I Will Throw You off Your Ship and You Will Drown and Die': Death Threats, Intra-Fandom Hate and the Performance of Fangirling", In. Booth, P. and Bennett, L. (eds), Seeing Fans: Representations of Fandom in Media and Popular Culture, London: Bloomsbury Academic

Kozinets, R. V. (2010). Netnography: Doing Ethnographic Research Online, London Sage: Publications

Kozinets, R. V. (2006). Netnography 2.0, In Belk, R. W. (ed.) Handbook of Qualitative Research Methods in Marketing, U.K: Edward Elgar Publishing:

Kozinets, R. V. (2002). The Field Behind the Screen: Using Netnography for Marketing Research in Online Communities, Journal of Marketing Research, 39, 61-72

Kramer, A. D. I., Guillory, J. E. \& Hancock, J. T. (2012). Experimental evidence of massive-scale emotional contagion through social networks, Proceedings of the National Academy of Sciences, 111(24), 8788-90.

MashableUK, (2012, October 26). \#BaldForBieber Hoax Teaches Kids to Fact Check Before Shaving Their Heads, [article] http://mashable.com/2012/10/26/bald-for-bieber/

Mayheu, M. M., Pulier, M. L., Wilhelm, F. H., McMenamin, J. P. \& Brown-Connolly, N. E. (2004). The Mental Health Professional and the New Technologies: A Handbook for Practice Today, USA: Taylor and Francis

McDermott, E., Roen, K. \& Piela, A. (2013). Hard-to-Reach Youth Online: Methodological Advances in Self-Harm Research, Sexuality Research and Social Policy, 10 (2), 125- 134

McDonald, K. E., Conroy, N. E. \& Olick, R. S, The Projects Ethics Panel (2017), What's the Harm? Harms in Research With Adults With Intellectual Disability, American Journal of Developmental Disabilities, 122 (1), 7892, 94,96

Metro (2016, August 1). In full: Chilling texts from girl who 'encouraged boyfriend to kill himself', [article], http://metro.co.uk/2016/08/01/in-full-chilling-texts-from-girl-who-encouraged-boyfriend-to-kill-himself$\underline{6042041 /}$

Nordahl, J., Beran, T. \& Dittrick, C. J. (2013). Psychological Impact of Cyber-Bullying: Implications for School Counsellors/L'effet psychologique de cyber-intimidation : Implications pour les conseillers scolaires, Canadian Journal of Counselling and Psychotherapy (Online), 47 (3), 383 - 402

PNAS (2014). Editorial Expression of Concern: Experimental evidence of massivescale emotional contagion through social networks, Proceedings of the National Academy of Sciences, 111(29), 10779.

Proctor, W. (2017). Fear of a \#Blackstormtrooper?: Hashtag Publics, Canonical Fidelity and the Star Wars Faithful, forthcoming

Proctor, W. (2016). A New Breed of Fan? Regimes of Truth, One Direction Fans and Representations of Enfreakment. In Bennett, L. and Booth, P., (eds) Seeing Fans: Representations of Fandom in Media and Popular Culture. London: Bloomsbury Academic

Rice, S., Robinson, J., Bendall, S., Hetrick, S., Cox, G., Bailey, E., Gleeson, J., \& Alvarez-Jimenez, M. (2016). Online and Social Media Suicide Prevention Interventions for Young People: A Focus on Implementation and Moderation, Journal of the Canadian Academy of Child and Adolescent Psychiatry, 25 (2), $80-86$

Richardson, B. G., Surmitis, K. A. \& Hyldahl, R. S. (2012). Minimizing Social Contagion in Adolescents Who SelfInjure: Considerations for Group Work, Residential Treatment, and the Internet, Journal of Mental Health Counselling, 34 (2), 121 - 132 
Rimm, M. (1995). Marketing Pornography on the Information Superhighway: A Survey of 917,410 Images, Descriptions, Short Stories, and Animations Downloaded 8.5 Million Times by Consumers in over 2000 Cities in Forty Countries, Provinces, and Territories, Georgetown Law Journal, 83, 1849 - 1934

Schneider, S. K., O’Donnell, L., Stueve, A. \& Coulter, R. W. S. (2012). Cyberbullying, School Bullying, and Psychological Distress: A Regional Census of High School Students, Journal of Public Health, 102 (1), 171 -177

Schrag, Z. M. (2011). The case against ethics review in the social sciences. Research Ethics, 7 (4), 120-131

Stern, S. (2004). Studying adolescents online: A consideration of ethical issues, in Buchanan, E. Readings in virtual ethics, Hershey and London: Information Science Publishing

Stewart, K. \& Williams, M. (2005). Researching Online Populations: The Use of Online Focus Groups for Social Research, Qualitative Research, 5, 395 - 416

Taylor, M. Quayle, E. (2003). Child Pornography: An Internet Crime, New York: Brunner-Routledge

Tumblr, (2016, June 23). Community Guidelines, [guidelines] https://www.tumblr.com/policy/en/community

Stewart, K. \& Williams, M. (2005). Researching Online Populations: The use of Online Focus Groups for social research, Qualitative Research, 5, 395 - 416

UK Council for Child Internet Safety (2017). UK Council for Child Internet Safety (UKCCIS), [guidelines], https://www.gov.uk/government/groups/uk-council-for-child-internet-safety-ukccis

Warrell, J. \& Jacobsen, M. (2014). Internet Research Ethics and the Policy Gap for Ethical Practice in Online Research Settings, Canadian Journal of Higher Education, 44(1), $22-37$

Whiteman, N. (2012). Undoing Ethics: Rethinking Practice in Online Research, London: Springer Science and Business Media

Wiles, R., Coffey, A., Robison, J. \& Prosser. J. (2010). Ethical Regulation and Visual Methods: Making Visual Research Impossible or Developing Good Practice?, Sociological Research Online, 17 (1)8, 1-10

Your Life Counts (2017). Know The Signs \& Symptoms To Prevent Suicide, [guidelines], http://www.yourlifecounts.org/learning-centre/know-signs-symptoms-prevent-suicide\#signs6

Zimmer, M. (2010). "But the data is already public": on the ethics of research in Facebook, Ethics and Information Technology, 12(4), 313-325. 